\title{
P-07 I7- Infusion of autologous-peripheral blood mononuclear cells: a new approach for limb salvage in patients with diabetes
}

Alba Di Pardo*, Enrico Cappello*, Giuseppe Pepe, Federico Marracino,Vincenzo Carrieri, Vittorio Maglione and Francesco Pompeo

Unit of Vascular and Endovascular Surgery - Division of Regenerative Medicine for Vascular and Neurovascular Diseases, IRCCS Neuromed, Pozzilli, Italy;

* The authors contributed equally to this work

Foot ulcer is a major complication of poorly controlled diabetes and probably the major component of the diabetic foot syndrome (DFS), which represents a very common precursor event prior of lower extremity amputation and the first cause of hospitalization in diabetic patients (Gde Dalem Pemayun et al., Diabet Foot Ankle 2015). Due to the multifactorial pathogenic profile, care of the diabetic foot often fails to sufficiently respond to conventional treatments such as drug thempy and vascular surgery. Over the last decade, research has mainly focused its efforts on developing cell-based therapies as potential alternative and more effective therapeutic interventions for skin regeneration and ulcer repair. Emerging evidence indicates that circulating mononuclear cells isolated from peripheral blood (PBMNC) have remarkable regenerative expansion capacities and are able to speed up tissue repair by promoting neoangiogenesis, collagen deposition and reepithelization in different pathological conditions (De Angelis et al., Tissue Eng Part C Methods. 2015; Kanazawa et al., Int J. of Mol. Sci 20I7).

\section{Aim}

The aim of this study is to further consolidate the therapeutic potential of autologous-PBMNC (A-PBMNC) infusion in diabetic patients with unresponsive skin ulcers, and to investigate the underlying molecular mechanisms.

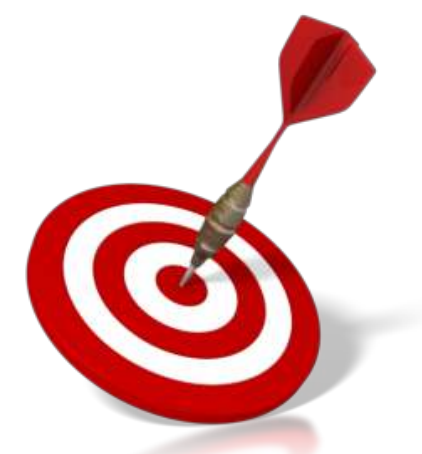

\section{Results}

Infusion of A-PBMNC in diabetic patients with skin ulcers leads to a clinically significant reduction in pain at rest and markedly accelerates the regeneration of damaged tissue and the overall healing of the wound (Fig 3.1 left). The beneficial effect of A-PBMNC infusion is also measured as ability of these approach to progressively reduce lesion size over the time (Fig. 3.I right).

Favourable clinical outcomes were integrated by histological evaluation of skin bioptic samples. Before treatment (T0) skin ulcer is hypoxic (Fig 3.2a), mainly characterized by enhanced inflammatory response (Fig 3.le) and unbalanced ratio between classically activated proinflammatory $(\mathrm{MI})$ and alternatively activated antiinflammatory macrophages (M2), in favour of MI polarization (Fig $3.2 \mathrm{~h}, \mathrm{~m}$ ), as well as by altered vascular homeostasis (Fig. $3.2 \mathrm{p}$ s).

Infusion of A-PBMNC promotes relevant changes in the overall molecular setting over the time ( $\mathrm{TI}$ and $\mathrm{T} 2$ ). The resulting cellular and biochemical adaptations favour the establishment of physiological-like conditions which progressively support the regeneration of damaged tissues and ultimately the wound healing (Fig 3.2 TI (b,c; f,g), T2 (i,l $\mathrm{n}, \mathrm{o})$ left; Fig 3.2 TI (q,r), T2 (t,u) right). As the regenerative processes occur, a graduale recovery of normoxia and increased vascular network formation is clearly seen.

\section{Material and Methods}

A-PBMNC were isolated from fresh venous blood sample through a new selective filtration and cell concentration system -WB Pall Celeris System (MonocellsCE marked under the 93/42/EEC Medical Devices Directive). After filtration, cells were infused back through multiple local, intralesional and perilesional intramuscular injections (see figure on the right bottom) and along the relevant axis. Molecular profile and biological responses to A-PBMNC infusion were investigated by histological examination of the incisional biopsy of the ulcers taken at different time points: before treatment (T0), 30 days after the first infusion (TI) and 30 days after the second infusion (T2)
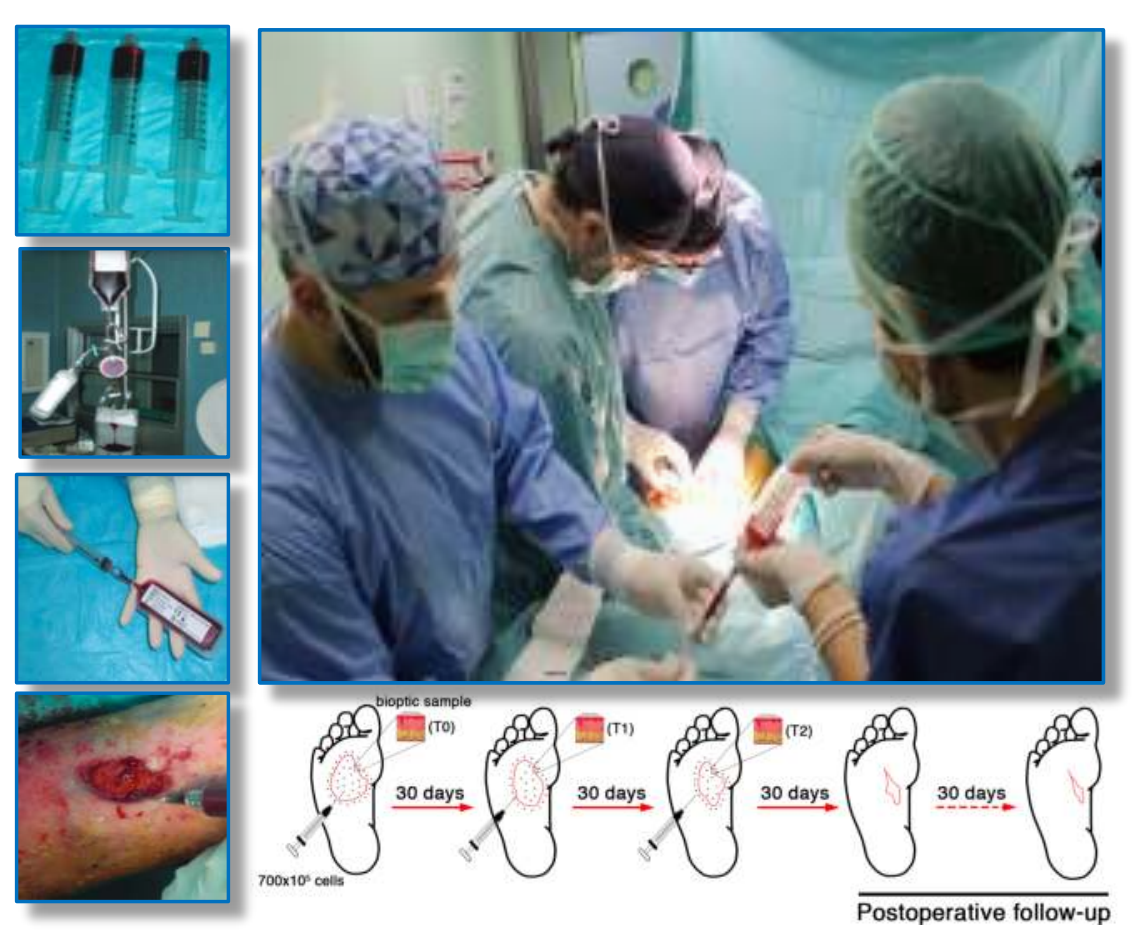

\section{I Infusion of A-PBMNC is critical for wound healing in patients affected with DFS}

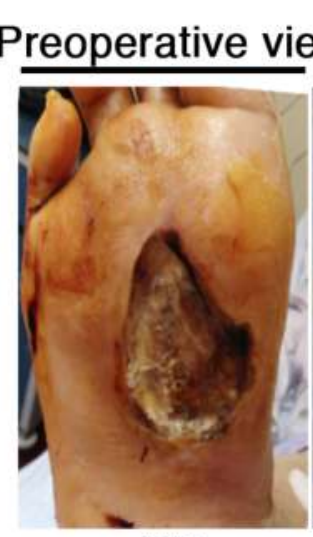

TO

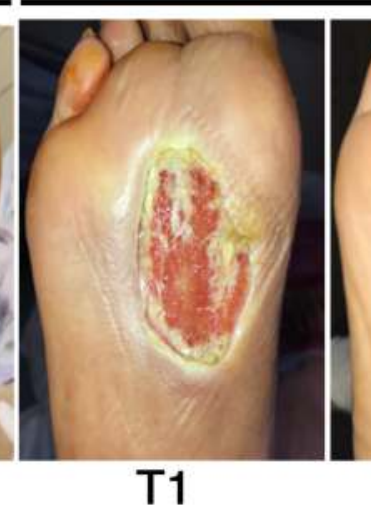

T1 Postoperative view

Fig. 3.I Representative micrographs showing preoperative and postoperative view of the skin lesion (left). Graph represents lesion size, normalized over the plantar area, before and after infusion (right).

\subsection{Infusion of A-PBMNC promotes molecular changes in favour of tissue regeneration}
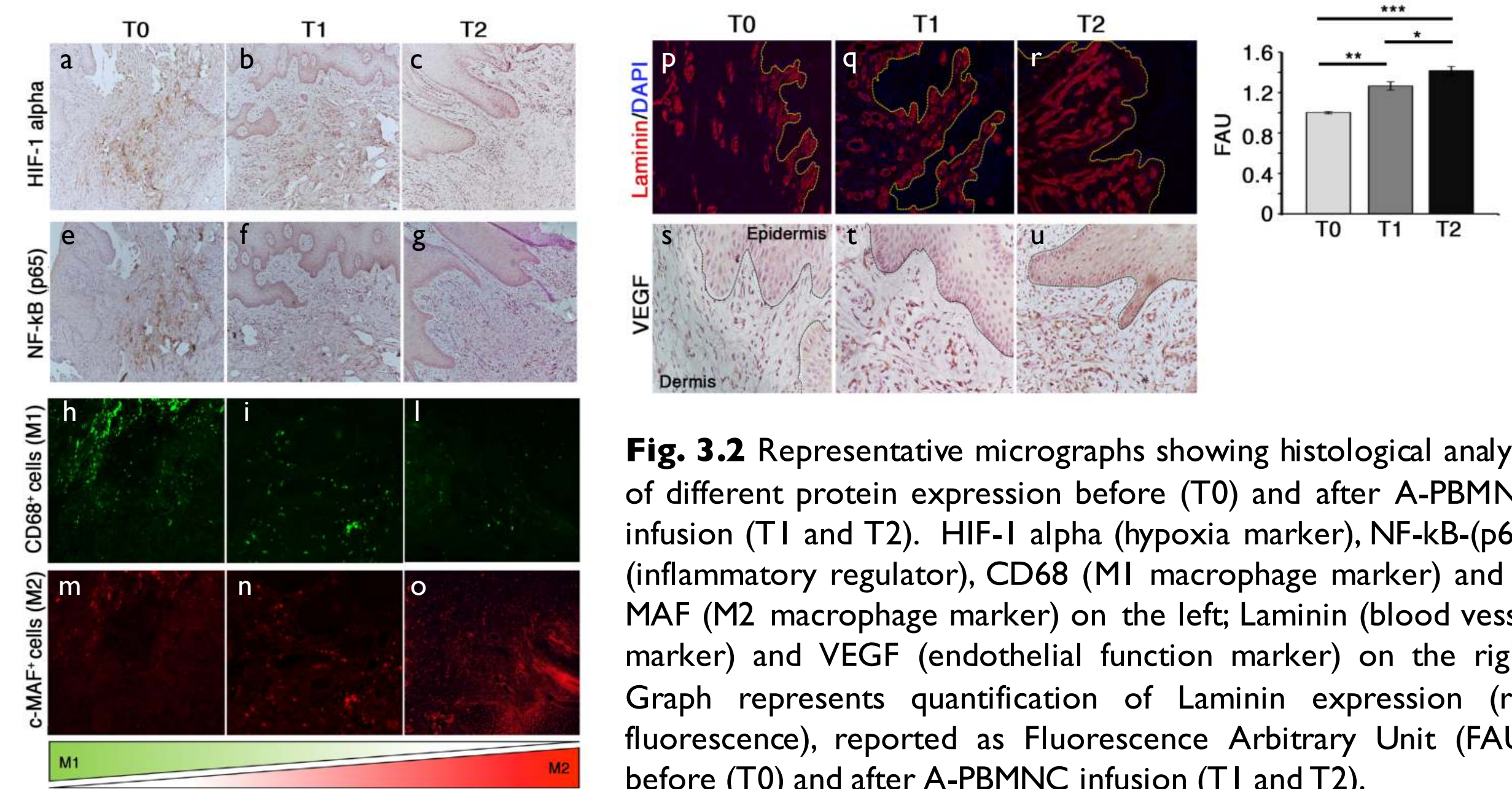

Fig. 3.2 Representative micrographs showing histological analysis of different protein expression before (T0) and after A-PBMNC infusion (TI and T2). HIF-I alpha (hypoxia marker), NF-kB-(p65) (inflammatory regulator), CD68 (MI macrophage marker) and cMAF (M2 macrophage marker) on the left; Laminin (blood vessel marker) and VEGF (endothelial function marker) on the right. Graph represents quantification of Laminin expression (red fluorescence), reported as Fluorescence Arbitrary Unit (FAU), before (T0) and after A-PBMNC infusion (TI and T2).

Conclusion. A-PBMNC-based therapy is an innovative and promising approach for the wound healing, regeneration of damaged tissues and tissue repair in DFS. Taking into account the prompt and long-term effects together with a favourable safety profile, it is plausible to believe that this strategy may significantly decrease the risk of amputation in diabetic patients with critical ischemia of the limb. From our perspective, all these properties make this procedure a key player in the future of the healthcare in such as chronic and worldwide disease. 